Rabaska

Revue d'ethnologie de l'Amérique française

\title{
L'Imaginaire des Grands Voyages indiens au Québec
}

\section{Louis-Edmond Hamelin}

Numéro 1, 2003

URI : https://id.erudit.org/iderudit/201604ar

DOI : https://doi.org/10.7202/201604ar

Aller au sommaire du numéro

Éditeur(s)

Société québécoise d'ethnologie

ISSN

1703-7433 (imprimé)

1916-7350 (numérique)

Découvrir la revue

Citer cet article

Hamelin, L.-E. (2003). L’Imaginaire des Grands Voyages indiens au Québec. Rabaska, (1), 43-59. https://doi.org/10.7202/201604ar d'utilisation que vous pouvez consulter en ligne.

https://apropos.erudit.org/fr/usagers/politique-dutilisation/ 


\title{
Terrains
}

\section{L'Imaginaire des Grands Voyages indiens au Québec}

\author{
Louis-EDMOND HAMELIN
}

Université Laval, Québec

Cette première étude d'un vieux sujet pose plusieurs questions : documentation, antériorité autochtone, types de déplacements vers l'intérieur, notion de Grand Voyage, localisation des itinéraires, genre de vie de ces voyageurs.

Le thème des plus longs circuits possède-t-il assez de documentation pour être l'objet d'une présentation ? Peut-on définir ces randonnées de bout du monde à partir de connaissances, d'expériences du terrain et de l'interrogation d'un passé possible ? Le cas échéant, comment à l'intérieur des terres distinguer les maxi-voyages du nombre incalculables de sorties, aussi braves mais plus brèves? Peut-on localiser quelques-uns des itinéraires suivant la trame des rivières, les portages (sentiers de transbordement) ponctuels, les limites des grands versants hydrographiques et la localisation des habitants? En outre, se pose le problème de la datation et du niveau d'autochtonité de ces immenses déplacements.

Il n'existe de sources écrites que pour environ $10 \%$ du temps de parcours et elles traitent surtout des voyages courts et proximaux. L'on peut cependant compter sur des travaux archéologiques, des archives des missions, des rapports de non-Autochtones ainsi qu'extrapoler à partir de la littérature orale, de la localisation des lots de piégeage, des sentiers de chasse ainsi que des sites de provision lithique. C'est peu.

Étant donné toutes les ignorances que la science ne semble pas prête à lever, l'étude des voyages de très longue durée apparaît plutôt vierge et répond à un certain vacuum. En conséquence, le présent texte emprunte beaucoup à l'imaginaire, mais un imaginaire qui ne devrait pas être trop éloigné des réalités du temps.

\section{TOUS TYPES DE DÉPLACEMENTS VERS L'INTÉRIEUR}

Dans la grande péninsule entourée d'eaux nordiques, les Autochtones occupent alternativement deux types de territoires : ils vivent soit agglomérés dans de modestes villages, soit dispersés dans les terres; dans le second cas, 
ils sont des Nutshimiu ${ }^{1}$ [Indiens de l'intérieur], mais ceux-ci peuvent l'être du proche comme du très lointain. De toute façon, qu'ils soient ici ou là, ils attestent d'une intimité millénaire avec leur milieu.

\section{Essai de classification des séjours sur le terrain}

Les très nombreux va-et-vient des Autochtones « dans les terres » peuvent être vus suivant leur documentation, motif, fréquence, âge et durée. Ils vont dans l'intérieur pour une grande variété de motifs identifiés comme alimentaire, commercial, familial, religieux, personnel, aventurier, militaire et politique.

Quant à l'âge, les grands canotages des Indiens méridionaux dans la vaste péninsule du Québec-Labrador se seraient déroulés en deux longues périodes : celle des millénaires qui ont précédé les découvertes, puis celle des centaines d'années postérieures.

Aire préhistorique - La première qui n'a pas laissé de littérature écrite est soumise aux découvertes archéologiques ; celles-ci peuvent suggérer l'échelonnement de grands déplacements au cours de plus de 3000 ans.

Aire historique - La seconde période, ethniquement plus complexe, est plus riche au plan de l'information. D'une part, existent les rapports produits par les non-Autochtones qui commencent à voyager sur les cours d'eau du Nord. Ces pénétrateurs sont des missionnaires comme le jésuite Albanel (1672), hommes d'affaires des compagnies de fourrure telle la Baie d'Hudson à l'ouest ou du Domaine du Roi à l'est, cinéastes comme Robert Flaherty, policiers, arpenteurs, prospecteurs et chercheurs, tels A.-P. Low, Jacques Rousseau ${ }^{2}$. Comme la plupart se font accompagner de guides indigènes, il faudra évaluer le sens de cette aide.

D'autre part, à partir des Découvertes, les Indiens continuent d'entreprendre tous types de voyages dont ceux d'opérations commerciales (Tadoussac) ou de rencontres politiques, tel le fait de se rendre à des traités de paix (Montréal, 1701). Pour leur part, les Iroquois du temps quittent l'archipel d'Hochelaga pour atteindre la Gaspésie, le haut Outaouais et la baie de James, la Hudsonie ou l'aire new-yorkaise. Outre les opérations précédentes, de très nombreuses autres, généralement d'amplitude familiale, correspondent à des sorties genre-de-vie, exigeant, elles aussi, un éloignement des villages; la durée de l'absence est variable, de quelques semaines à quelques mois, à deux hivers à l'intérieur de dix-huit mois, enfin, à deux ou trois ans. On fait le voyage d'une seule traite ou par étapes. Certains migrants ne reviennent pas par décès ou intégration à une autre communauté.

1. Les entités en italique sont des autochtonymes (ceux du Québec portent référence aux Indiens et aux Inuits).

2. Jacques Rousseau, À travers l'Ungava, Montréal, Mémoires du Jardin Botanique de Montréal, $n^{\circ} 4$, 1949, pp. 83-131 (pagination de la revue L'Actualité économique). 
Théoriquement, au tout début, on navigue sur le même cours d'eau; puis, on utilise tous les affluents d'un même grand collecteur, évolution qui rappelle le système de la cueillette des fourrures à partir de la baie de James ou à l'intérieur du Domaine du Roi dans l'arrière Côte-Nord. Plus on s'éloigne, plus on a de chances de sortir des bassins hydrographiques d'origine ; à la fin, on quitte les hauteurs des terres laurentiennes et hudsoniennes pour pénétrer dans les bassins arctique et atlantique-nord. Un tel enjambement exige de longs portages comprenant l'identification du ruisseau de faible pente qui débouche, c'est-à-dire conduit à un vrai maskino ou chemin coulant.

Une certaine documentation écrite ${ }^{3}$ existe à laquelle s'ajoute le secours d'une littérature orale non abondante ${ }^{4}$; au hasard, s'y joignent des notes éparses dans les archives des missions ainsi que dans quelques journaux de voyage.

\section{Préparation}

Les peuples bien adaptés au Nord ne réagissent pas de la même façon que la très grande majorité des gens du Sud ; les premiers préfèrent les aires qui apparaissent les plus difficiles aux seconds, à savoir une vaste étendue, sans chemin terrestre, parsemée d'arbres, de lacs, de rivières et d'animaux sauvages, quand ce n'est pas durant le plein hiver. Pour les nordistes, l'immense péninsule, apparemment uniforme et confuse, se présente comme un écoumène infiniment varié et ouvert au transport, à l'habitat et à la chasse. On peut vivre entièrement du pays. Les milliers de plan d'eau sont autant de maskino, ces chemins coulants gratuits qui deviennent comme allongés par les longs et petits portages qui les relient. Réservoir naturel, ce milieu offre, entre autres, du bois de chauffe ou de construction, des eaux de boisson, de cuisson ou d'ablation, des animaux de trappe, de nourriture ou de couverture ; la volaille se prend à même les volées de passage ou aux atterrissages de la sauvagine trompée par l'invitation d'intimité des appelants. Pour les gens des bois, un

3. José Mailhot et A. Michaud, North West River: Étude ethnographique, Québec, Centre d'études nordiques, «Travaux divers » $n^{\circ}$ 7, 1965,120 p. Les auteurs utilisent l'expression de grandes migrations pour exprimer les déplacements des Indiens entre la Moyenne Côte-Nord et le Labrador central (environ 700 kilomètres de canotage dans un sens); les déplacements se font, antérieurement à 1950, pour la recherche d'un conjoint, la reconstitution familiale, la pratique des sacrements (baptême) et le goût de l'aventure. Gérard Duhaime (dir.), Atlas historique du Québec. Le Nord, Québec, PUL et Gétic, 2001, $207 \mathrm{p}$. : en particulier les articles de Yves Labrèche, Paul Charest, François Trudel et B. Saladin d'Anglure. Les migrations étudiées dans ces publications de 1965 et 2001 se présentent comme intermédiaires entre les très nombreuses courses usuelles en forêt et les rares Grands Voyages du Nord dont parle surtout le présent article.

4. L'auteur possède quelques expériences de sections au long de quatre des itinéraires. Au cours d'un déplacement nordique d'il y a plus de trente ans, il entend même une grand-mère indienne de la Côte-Nord raconter son voyage de noces au lointain Mushuau Nipi, site emblématique du Grand Voyage du Nord. 
tel environnement est source de petits bonheurs sans cesse renouvelés ; les Indiens des grands espaces ne se sentent éloignés de personne; s'ils ne disposent pas de tout, ils ont l'impression de ne manquer de rien.

Pour tout voyage, la préparation n'est pas une petite entreprise car elle exige imagination et expérience. On ne part pas pour faire des courses sportives, sauter le rapide le plus dangereux et raccourcir le trajet ; on sait d'expérience qu'il faudra prendre le temps de faire des zigzags, en l'occurrence accomplir plusieurs fois des déplacements vers le sud afin de trouver une bonne passe vers le nord; les Anciens conseillent même d'entreprendre des détours afin de profiter des meilleurs endroits pour la cueillette de nourriture et l'échange d'amitiés avec des familles également en transit. Dans ces conditions d'humanité, les bonnes voies n'allongent pas.

La liste des objets ne doit rien oublier d'un essentiel qui ne pourrait être produit là-haut, cueilli dans la nature, échangé sur place, repris dans des caches ou acheté à des comptoirs. Il faut rassembler canots en très bon état, tentes, matériel de cuisson, gros sel, gros chaudron, bassine, couteaux, grattoirs, chandelles, aiguilles, lames de scie, haches, pierre à aiguiser, poinçons, poêles et bouts de tuyau, pièges pour petits gibiers, dards, fils assez résistants pour cordeler (haler) le lourd canot de charge à partir d'un sentier de halage le long des rapides qui ne se sautent (canoter) pas. Parfois, on apporte un pot de peinture pour rajeunir les inscriptions en syllabique du nom des personnes décédées au cours de voyages précédents.

On emballe les articles dans des paquets qui tiendront bien les rapides à sauter et qui, dans les autres cas, seront transportables à dos d'homme dans le sentier du portage; paquetage et portageage ${ }^{5}$ correspondent à deux opérations très liées tellement il faudra souventes fois charger et décharger les embarcations. En tout temps, on doit protéger les pièces qui ne peuvent prendre d'eau, comme les fusils, les munitions, la farine pour la banique $e^{6}$, les allumettes, des aliments de secours, des plantes de guérison, du thé et la torquette de tabac. Lors de chaque transbordement et pendant des centaines de fois, on devra faire attention à n'oublier aucune pièce. La logistique est donc exigeante. Une certaine compensation des efforts exigés par chaque portage viendra de la prise de gibier ainsi que de la rencontre de familles également migratoires ; avec elles, fonctionne aussitôt l'attendu mocassintélégraphe (moyens autochtones d'une diffusion étonnamment rapide des messages) qui fait parler de chacun, à la satisfaction de tous.

5. Canadianisme. Fait de canotiers et passagers de marcher dans un petit chemin tortueux périphérique à un cours d'eau, vers l'amont ou vers l'aval, avec charge, en vue de reprendre la voie fluviale au moment où elle redevient navigable. On fait aussi du portageage d'une nappe d'eau à l'autre.

6. Galette de farine délayée, cuite sans levain et de forme arrondie. 


\section{Camp principal}

Généralement, au cours d'un voyage de quelques années, les Indiens établissent une habitation plus confortable où ils passeront quelques mois, voire plus d'une année. Un grand soin accompagne cette opération majeure. Pour le choix du site, tous les traits naturels de chaque saison doivent être considérés. Le lieu doit être ensoleillé, facile d'approvisionnement en bois debout pour le chauffage et pour un peu de construction, en produits de pêche fluviale de même qu'en eau de tout usage. L'idéal est de se trouver près d'un mont qui protège du vent et à l'ombre duquel on installe des caches de nourriture, en cas de redoux ou d'accidents chez les chasseurs. Par famille, on monte une grande tente si possible pontée, à savoir possédant un plancher de bois sur lequel on étendra des peaux remplaçant le substrat des croix de sapin; on élève un mur de bois, doublé également de fourrures, jusqu'à mi-hauteur de la tente, ce qui fournit une surface en dur en vue de l'installation de crochets et tablettes; on double le toit afin d'emprisonner une chambre d'air isolante tout en essayant de récupérer un peu de la chaleur qui se concentre au sommet du logis ; on amoncelle de la neige face à la porte extérieure afin de contrer le vent. À ces constructions mi-bois, mi-tissus et peaux, s'ajoutent des installations secondaires pour le séchage des cuirs, le fumage du poisson, la suspension d'articles à protéger de même que le support des canots ou des traînes ; pour isolement et secours, un tipi (tente conique) à feu ouvert est dressé ; il existe aussi un lieu d'aisance ainsi qu'un abri, l'hiver, pour empêcher d'avoir à déneiger tous les articles après chaque tempête et, l'été, pour les tenir au sec. Après quelques semaines, on aura élevé tout un petit hameau fonctionnel qui rappelle le village coutumier d'en-bas. On a conscience de vivre comme chez soi ; les enfants dorment dans la douceur des peaux de lièvre ; on cultive des sentiments de proximité, d'intimité et de ferveur à l'endroit du territoire, comportement sans pareil chez les non-Autochtones.

La production d'aliments constitue une occupation irremplaçable. Aux produits frais, s'ajoutent du poisson et de la viande, boucanés et séchés. Les activités de chasse, peu préoccupées de hiérarchie, renvoient à un système de complémentarité et de partage ; l'effort de chacun répond à un objectif au service de tous. Dans les temps morts, les adultes complètent la formation des jeunes à la vie en forêt, fabriquent des raquettes ou des lames pour les traînes, coupent de fines et longues perches pour consolider les habitations. Les femmes s'affairent à la préparation de la nourriture, à la finition de la fente du bois de chauffage, à l'éducation des enfants, à l'équarrissage des peaux ainsi qu'à la couture des mocassins ; elles s'occupent aussi de suivre le quantième afin de faire réciter, le dimanche même, les prières du dimanche. 
Les garçonnets s'affairent, l'hiver, au déneigement des toits et au nettoyage des tuyaux alors que, l'été, à un consciencieux entretien des canots; en toute saison, ils changent la litière coniférienne et font de la petite chasse. Les fillettes s'occupent des enfants, en plus de participer à de légers travaux domestiques; l'été, elles s'amusent à déposer des petits cailloux dans les sections les moins sèches des sentiers environnants. Chacun et chacune sont fort occupés, suivant la tradition.

\section{L'antériorité des Autochtones}

Les canotages nordiques posent la question de l'identification des opérateurs initiaux ; ont-ils été autochtones ou non autochtones? L'argumentation théorique apporte un premier éclairage. Si les étrangers au territoire devaient obligatoirement utiliser des guides indiens comme pilotes, alors le service irremplaçable de ces derniers fournirait la preuve que les Grands Voyages préexistaient à la colonisation; la conclusion serait inverse si les nonAutochtones eux-mêmes avaient plutôt donné aux Indiens l'idée d'entreprendre des déplacements transpéninsulaires; dans ce cas, la participation des résidents serait réduite à celle d'hommes à tout faire et cacherait les mérites de toute antériorité.

Mais l'antériorité amérindienne est confirmée par l'archéologie. L'occupation autochtone de l'intérieur de la péninsule, bien datée malgré ses milliers d'années, n'aurait pu se faire sans de longues navigations comportant portages et campements ; de plus, les artéfacts lithiques prouvent à un niveau indiscutable l'existence de longs transports de matériaux rares à partir de toutes les rives vers l'intérieur de la péninsule, et vice-versa. De temps immémoriaux, les Autochtones naviguent sur les autoroutes fluviales. À ce sujet, l'incertitude n'existe pas.

\section{NOTION ET EXEMPLES DE LONGS VOYAGES}

Des familles des communautés villageoises entreprennent au moment opportun des parcours pluriannuels; l'un d'entre eux est désigné Grand Portage ou Mista ka pitagan 7 . Par définition, un Grand Voyage correspond à une migration à long cours pouvant atteindre 5000 kilomètres aller-retour et comportant canotage, marche, de nombreux campements itinérants et une (ou deux) installation de moyenne durée. Chez les non-Autochtones, de tels

7. L'expression est dans H.Y. Hind, Explorations in the Interior of the Labrador Peninsula: The Country of the Montagnais and Nasquapee Indians, London, Longman, 1863, 2 vol. Mention de ce Grand Voyage sur une carte, L.-E. Hamelin, Passer près d'une perdrix sans la voir, Montréal, McGill, PEQ, 1999, p. 63. Sotrac, Uemashtan, le Grand Portage. Sept-Îles, 1995, vidéo, 16 minutes. Commission des droits de la personne, Mythes et réalités sur les peuples autochtones, Montréal, 2002, carte de piégeage : p. 65 . 
déplacements d'amplitude vers des endroits comportant des surprises sont des exploits désignés du nom pompeux d'expéditions.

\section{Le concept de base}

Le Grand Voyage inclut certes le petit espace pédestre situé entre deux courtes navigations - désigné portage - mais il est loin de s'y limiter; il ne renvoie pas non plus à un unique portage qui serait seulement plus difficile par sa longueur, sa pente, son encombrement végétal, son mauvais drainage ou sa faible productivité en produits de chasse locale. Il correspond plutôt à un tout autre type de voyage, un voyage d'envergure, nécessitant un éloignement prolongé du domicile permanent de même que la gestion de risques inhabituels et imprévus. Il s'agit d'une traversée circulaire qui, après des années, fait revenir les participants au point de départ. Pour des questions de santé, d'équipement et de temps disponible, peu de familles d'Indiens du Sud sont en mesure de se rendre jusqu'aux confins septentrionaux de l'immense péninsule. Un Grand Voyage est donc un événement plutôt rare.

\section{Cinq itinéraires}

En fonction du bassin du Saint-Laurent, on dénombre cinq principaux circuits dont chacun traverse la hauteur des terres en direction soit de l'ouest, du nord ou de l'est. Ces impressionnants trajets sont énumérés d'ouest en est.

A- Le circuit occidental relie Montréal à la baie de James par l'Outaouais et l'Harricana.

B- Afin de réaliser celui, ou plutôt ceux, orientés SE/NO, on remonte le long affluent Ashuapmushuan du fjord Saguenay, ce qui permet d'atteindre un point de bifurcation: $\mathrm{Ba}:$ l'une des voies mène au Mistassini ${ }^{8}$, lac providentiellement situé à mi-chemin entre la haute Côte-Nord et la Radissonie hudsonienne ; puis, le grand lac, par la rivière Rupert, se déverse dans la baie de James ${ }^{9}$; Bb : 1'autre fourche passe par le lac Matagami et la rivière Nottaway pour se jeter au sud de la même baie.

C- Le plus difficile des cinq déplacements relie le Saguenay à la baie d'Ungava en empruntant principalement les rivières Péribonka et Caniapiskau. Comme le voyagement suivant, l'axe $\mathrm{C}$ atteint le Grand Nord ou l'Arctique.

8. Albanel, lac jumeau, peut jouer le rôle d'un lac de shamans où se trouve une TchichéManitou Ouitchchouap [grand-esprit-maison], tel que défini par le père Pierre Laure, Carte du Domaine en Canada dédiée à Mgr le Dauphin, Chekoutimi, 23 août 1731 (original à Paris). Cette carte a connu diverses versions comportant des variantes. Dans la vaste région lacustre, se trouvent des pierres recherchées par tous les Autochtones d'avant les Découvertes, tels le quartzite, le chert et le jaspe.

9. D'après un conte, les Indiens de la baie de James se font une image des Indiens de l'Atlantique ; sans des déplacements transpéninsules, une telle création culturelle ne serait pas probablement pas née. 
D- Le voyage très symbolique qui suit est moins fréquenté que les portages allant vers l'Hudsonie mais davantage que le précédent; il relie le bassin laurentien par les cours d'eau Moisie, Nipissis, Ashuanipi, Menihek, Attikamagen et George au royaume mythique du caribou dont le site emblématique est le [lac] Mushuau Nipi.

E- Enfin, le cinquième itinéraire rejoint l'Atlantique, c'est le Grand Voyage de l'Est liant plusieurs cours d'eau de la Basse Côte-Nord au Labrador intérieur, puis du Labrador côtier.

Par analogie, les ambitieux trajets commerciaux en canot à partir de Montréal vers le lac Supérieur et au-delà ont les attributs des Grands Voyages autochtones à l'intérieur du Québec péninsulaire.

Ces cinq itinéraires schématiques ne doivent pas voiler un nombre considérable de variantes locales et de longs raccordements d'un axe au voisin ; ainsi, entre A et $B$, on peut passer de l'Harricana au Matagami, entre $\mathrm{B}$ et $\mathrm{C}$ faire le pont entre le lac Mistassini et la Péribonka ou la Mistassini, entre $C$ et $D$, utiliser la Sainte-Marguerite en aval, traverser le lac Pipmuacan et, près du coude de la Caniapiskau, le lac Wakuach, enfin entre D et E, rejoindre la côte atlantique par le fleuve Churchill au centre ou par l'Assiwaban à l'est du Mushuau (voir CARTE).

Le dit toujours un peu vantard des hommes de canot est confirmé par le témoignage de grands-mères des Réserves qui ont enfanté dans les profondeurs de l'ouest, du nord et de l'est de la péninsule. Pour sa part, un saint-curé missionnaire, dès son arrivée dans la voie $\mathrm{Bb}$ du Moyen Nord forestier, avait été étonné d'apprendre que le voyage indien de noces, l'apampiyish $k a$ nishikaboch ${ }^{10}$, était aussi le fait de quelques ménages qui partaient joyeux pour des « finisterres » bien lointains.

Ces cinq trajets sont seulement quelques exemples des nombreuses traverses trans-Québec qui répondent à un réseau très développé de cours d'eau navigables.

10. L.-Ph. Vaillancourt, Dictionnaire français-cri, Québec, Presses de l'Université du Québec et Fondation UQAC, 1992, p. 459. 


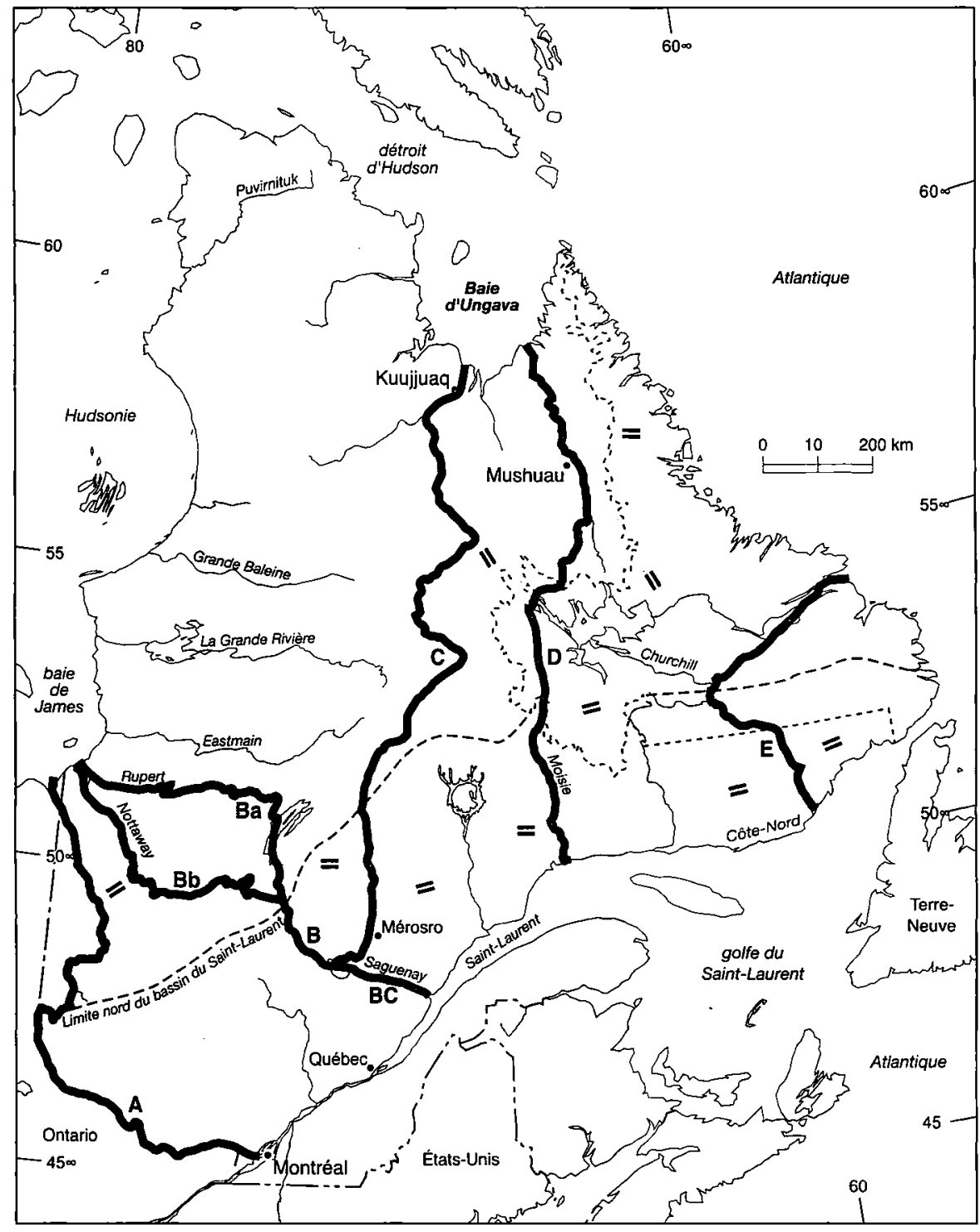

L.E.H. 1522

Tracé schématique de cinq Grands Voyages joignant le Saint-Laurent ${ }^{11}$

11. Voir le texte pour une description des voies A, B, C, D et E. Par le même Saguenay, les voyageurs s'engagent à la fois dans les circuits $\mathrm{B}$ (dont $\mathrm{Ba}$ et $\mathrm{Bb}$ ) et $\mathrm{C}$. Les deux tirets rapprochés indiquent quelques raccordements d'un itinéraire à l'autre. 


\section{Le Grand Voyage du Nord, annÉEs 1930}

Trois familles indiennes de Mérosro ${ }^{12}$ choisissent le Grand Voyage du Nord. C'est la voie D par le difficile raccordement Pipmuacan de la voie C.

\section{Départ}

La tradition orale aide à répondre au premier problème, celui de la date de départ qu'on établit en comptant à rebours. On fixe d'abord le moment souhaitable d'arrivée là-haut, c'est-à-dire au Mushuau Nipi [nipi, lac]. À ce haut lieu, on doit être au plus tard au début de septembre afin de monter le campement de même que de ramasser petits fruits et végétaux avant la froidure hivernante ; cette fois, une raison supplémentaire est fournie par le fait que l'une des femmes est enceinte en vue d'un accouchement prévu pour le début d'octobre. Tôt faut-il quitter Mérosro même si, au printemps, les hautes eaux du Moyen Nord ont beaucoup de tirant et que la terre n'est pas complètement libérée de bancs de neige et de plaques glacielles ${ }^{13}$; aussi dans certaines sections, pourra-t-on faire glisser les canots une fois mis sur support, même sans les décharger. On partira donc de la Réserve au début de mai en prévoyant un déplacement moyen de quelques kilomètres par jour ; pour cela, il faudra éviter maintes occasions de prendre du retard.

À l'aller, on se déplace de biais vers le Nord-Est, contre le soleil, le matin, et dans son prolongement, le soir, situations d'éclairement utiles dans l'orientation générale des voyageurs. De Mérosro, le parcours de travers nécessite de gérer de très nombreux rapides avant de rejoindre une chaîne de lacs de bonne navigation et dont les désignants se terminent par les assonances culturelles an, ane, ani, anie. Une fois sorti des limites septentrionales du bassin du Saint-Laurent, on se déplace sur un plateau central comportant d'autres nappes lacustres étendues, cette fois désignées par une finale en $a u$. Par temps calme, afin de traverser en sécurité ces vastes lacs, on se lève très tôt dans l'espoir d'arriver sur la rive d'en face avant la montée d'un vent qui se produirait au milieu du jour ; parfois, plusieurs tentatives sont nécessaires au succès de ce genre de déplacement. Au total, les étendues lacustres et les eaux mortes font de $30 \%$ à $40 \%$ de l'itinéraire total.

Au-delà, des ruisseaux s'écoulant vers le Grand Nord encouragent l'équipe.

Les Nutshimiu de Mérosro comprennent huit personnes dont quatre viennent de la famille Simon et Catherine Wawati, trois de la famille Benjamin et Esther Shécapio de même que le veuf sans enfant Paul Bellefleur, soit cinq adultes, un garçon de seize ans et deux enfants, tous Indiens en bonne santé.

12. Les noms des individus de même que les toponymes Camp du Caribou, Lac des Shamans, Mérosro (Réserve) et Djouac-jjuaq (grand-grand ; écoumène inuit) sont fictifs, mais vraisemblables.

13. Glaciel, néologisme, Québec, 1959. Dans les Petit Larousse et Grand Robert. 
La flottille rassemble quatre canots dont deux canots de famille, un canot de secours et un canot de charge ; seul ce dernier, conduit par Paul, peut compter sur un petit moteur qui sera utilisé jusqu'à l'épuisement du carburant ; on devrait atteindre ainsi le plateau central où l'engin, devenu techniquement inutile, sera mis sous cache jusqu'au moment de le reprendre au retour, on ne sait pas quand ; les barils d'essence, maintenant tous vides et une fois nettoyés par les eaux et le sable abrasif d'un rapide, sont utilisés comme contenants.

Au village, le départ comme tel fait naître une cérémonie d'émouvantes amitiés. Toute la communauté est présente, les uns regrettent ne pas être encore d'un Grand Voyage ; d'autres n'osent pas manifester leur crainte, des amis ne sont-ils pas décédés là-bas ? Des enfants regrettent de s'éloigner de compagnons de jeux. On regarde discrètement, mais on regarde bien les aventuriers, comme si on pouvait ne jamais les revoir. Tous les canots du village accompagnent la petite troupe jusqu'au premier rapide où le saint-curé la bénit ; les trois meilleurs canotiers se font des accompagnateurs jusqu'au premier grand lac dont le nom se termine en an, question de participer au portageage, hâter l'arrivée de l'équipe au lointain Mushuau et saluer des amis riverains.

\section{Le paysage topographique}

Les membres qui en sont à leur première pénétration dans le Nord se font expliquer le phénomène du tissékau, montagne à versant dissymétrique. Il s'agit plutôt d'un sommet montagneux qui marche. L'illusion se produit lorsqu'un observateur, sis en contrebas d'un abrupt à dôme herbeux, voit ce dernier comme modifié par l'agitation saccadée de caribous panachés qui broutent au sommet. Cet extraordinaire événement n'est pas que visuel car il porte pour les familles-témoins, localisées au piedmont, la promesse assurée de bons repas comportant bouillon, moelle et viande dont elles rêvent de consommer en grand depuis des années.

Au fur et à mesure que l'on avance vers le Nord, le paysage parle de plus en plus du caribou, animal mythique qui, en fait, est le motif des grands voyages des Indiens du Saint-Laurent vers l'Arctique ; ici et là, ils voient des sentiers bien creusés sis au sommet des collines serpentives dénommées $e^{e s k e r^{14}}$, des panaches égarés et des écheveaux de toison accrochés aux arbustes. Puis, les familles de Mérosro arrivent enfin au prestigieux Mushuau Nipi ou lac des $《$ terres sans arbres $»^{15}$, suivant une locution caractéristiquement expressive, même s'il faudrait plutôt entendre « terres sans forêt »; la vraie terre sans arbre est la toundra, déjà présente au sommet des tissékau.

14. Colline de cailloux.

15. Louis-Edmond Hamelin, Le Mushuau Nipi à l'âge du caribou, Québec, Centre d'études nordiques, «Nordicana » $n^{\circ} 36,1973,107$ p. Préface de Pierre Perrault. 


\section{L'AN I}

Le groupe n'a pas établi d'office le nombre d'années à passer loin du village d'en-bas, les circonstances commandant le déroulement de la vie. L'année I nécessite une préparation minutieuse à Mérosro suivie d'un pénible canotage vers le nord, enfin, l'installation d'habitations de longue durée sur les rives du lac choisi. La naissance attendue chez Esther Shécapio se produit au moment prévu, soit après l'établissement du Camp du Caribou, mais la grande joie de l'événement est ternie par des difficultés imprévues lors de l'accouchement ; on devra donc prendre grand soin du bébé et de la mère.

En revanche, au début de l'automne, c'est la traversée, à une passe du lac très allongé du Mushuau, d'une horde interminable de milliers de caribous quittant le nord-est de la péninsule en vue de la grande forêt protectrice sise au sud-ouest. Jamais de mémoire d'histoires de chasse, il n'avait été question d'une promenade animale de cette envergure. Le miracle s'est produit tout comme le shaman l'avait prédit à Simon Wawati à la Ouitchchouap (maison) fréquentée par le TchichéManitou, près du lac Mistassini d'en bas. Aux yeux de témoins plus qu'émerveillés, les caribous sont si serrés en nageant qu'ils pontent le Mushuau Nipi par la construction d'une surface solide sur laquelle on pourrait traverser à pied ! Leur évaluation romanesque ne tient cependant pas compte du fait que le marcheur imaginé devrait difficilement enjamber un sous-bois d'andouillers fermes, courbes et en mouvement.

Le mythe des grands troupeaux répond donc à une réalité dure. La région de Mérosro pourtant giboyeuse est très loin d'offrir des cheptels d'une telle ampleur. Ce royaume nordique est bien la résidence de l'Atihknapéo ou Dieu du caribou dont parlent des légendes mais auxquelles on avait tendance à ne plus croire. Le Mushuau Nipi correspond au sanctuaire du caribou. À l'équipe des voyageurs indiens, la vue d'une telle foule d'animaux fait aussitôt oublier toutes les pénibilités endurées durant la venue. Quelle chasse miraculeuse ne fera-t-on pas après avoir destiné aux animaux les prières rituelles! Que de mokoshans délicieux, les réserves d'une viande bien conservée ne vont-elles pas assurer pour les mois de saison froide! Que de merveilles à raconter à Mérosro, se promettent Simon, Benjamin et Paul sans avoir à en inventer un peu cette fois !

Au Mushuau, l'abondance des cervidés du premier automne est à l'origine du faste de la deuxième année. Bien nourris et emmitouflés, les WawatiShécapio-Bellefleur y passent le plus grand hiver de toute leur existence. De plus, la vie est marquée par l'heureux accouchement par Catherine d'une fille qui, comme sa cousine de six mois, a l'honneur de naître au cours d'un Grand Voyage. La chasse pour la fourrure participe à la qualité du séjour ; en bonne quantité, des lynx, martres, visons, loutres, renards, castors, même des 
carcajous, pris au piège, vont servir à préparer des ballots de peaux roulés que l'on ira livrer au comptoir commercial du Grand Nord ; il serait encombrant d'avoir à transporter ces produits de trappe jusqu'aux postes du Sud.

En effet, à une centaine de kilomètres au nord du Mushuau se trouvent les territoires froids parcourus par le Peuple du phoque (Inuit) ; de tradition, Peuple du castor (Indien) dans la taïga et Peuple du phoque dans la toundra pratiquent généralement des rapports de distance. Pour éviter tout affrontement, le petit groupe indien de Mérosro, bien éloigné de sa base protectrice, a élevé son Camp du Caribou dans un lieu ordinairement non parcouru par les résidents de l'Arctique. En fait, la distance est non seulement ethnique mais écologique. L'Indien trouve hostile l'écoumène du Grand Nord qui ne lui offre pas ses repères coutumiers de chasse à l'abri d'un arbre ; sans cet ombrage, il est à découvert; l'Indien ne possède pas non plus la technique de tuer le morse en mer ni le goût de manger sa graisse ; dans une zone sans arbres, il s'inquiète des réserves de bois de feu et ne saurait comment fabriquer les petites clôtures de bois autour des stèles funéraires ; l'ours blanc, contrairement à l'ours noir des forêts, lui fait terriblement peur ; l'iglou est comme étranger à sa culture ; il n'a aucune expérience de la nuit polaire, même durant quelques jours. Bref, l'image sévère que les Indiens du Sud se donnent du pays et des gens du phoque les pousse à ne pas généralement dépasser vers le nord le bassin du Mushuau où se trouvent encore des bosquets amis. Dans le Grand Nord, on ne s'aventure que pour affaires, visant précisément un comptoir de traite qui échange des peaux de fourrure contre des articles d'un magasin général de première nécessité ; ce commerce, dirigé par des non-Autochtones, se trouve à assurer la normalité des opérations et une paix interethnique d'office protégeant et les uns et les autres.

\section{L'AN II}

Au cours de l'été et après l'arrivée annuelle au détroit d'Hudson du navire de ravitaillement gouvernemental (opération dite Sea Lift), l'équipe décide justement d'envoyer au comptoir commercial de Djouac-jjuaq l'adulte Simon Wawati avec le jeune homme Shécapio. Le but avoué du voyage est d'échanger les belles peaux bien grattées au Camp du Caribou contre des balles de fusil, de petits articles ainsi qu'un quart de fleur (tonneau étanche de farine) en vue de la fabrication de la banique dont on se prive depuis des mois.

En descendant la rivière, les deux canotiers, chargés comme on le sait, s'arrêtent quand même à une station de recherches désignée Finn, tenue par des non-Autochtones et dont les guides sont des Innus (autres qu'Inuits) frères du Labrador océanique. Grâce à leurs guides qui facilitent contacts et discours, les étrangers tiennent à recueillir d'autres informations au sujet des 
Grands Voyages, en l'occurrence ceux du Nord qui traversent la péninsule dans le sens des longitudes. Les gens de Mérosro acceptent la proposition ; sur le champ, l'organisation scientifique leur promet des objets pratiques qui font envie et leur avance quelques dollars devant être utiles au magasin du Grand Nord. Environ trois semaines plus tard, les deux Indiens de Mérosro arrêtent tel que promis à la station Finn et font un petit cabanage (campement pour séjour) afin de se prêter à la curiosité des gens de sciences. En échange de renseignements de première main, ils acceptent des articles comme une hache à fendre du gros bois, des agrès perfectionnés de pêche, des boîtes de conserve, un chiot pour amuser les enfants de même que deux petits bijoux à offrir à Catherine et Esther.

La visite au Grand Nord des deux Indiens du Camp du Caribou dure quatre semaines durant lesquelles rien d'autre de remarquable ne se produit. La peur mythique de se faire automatiquement voler par les gens du Peuple du phoque, qui traditionnellement fournissent une bonne excuse à revenir bredouille ou à perdre un objet, ne reçoit, cette fois non plus, aucune confirmation ; les Indiens prennent cependant toutes les précautions dans les longs sentiers tortueux où le matériel en transit doit être souventes fois déposé et repris.

Au camp de base du lac Mushuau, les beaux jours et les belles nuits favorisent la bonne vie et l'apprivoisement gratifiant de la nature. L'épouse Catherine, la seule du groupe à suivre fidèlement le calendrier, peut identifier le jour du dimanche dont une partie de la matinée est consacrée à la lecture du livre de prières, ce qui correspond par le fait même à un exercice de syllabique que chacun prolonge par l'écriture de quelques phrases.

En référence à l'alimentation, si la fin de l'année I avait été extraordinairement bonne, celle de l'année II est décevante. D'abord, l'automne est maussade par ses types de temps cassés, c'est-à-dire non soutenus, circonstances défavorables aux plans de chasse à l'échelle des jours. Au cours de ce pré-hiver ou pitci-pipon, ce n'est pas au Mushuau Nipi, mais probablement loin de cet endroit attendu, que les caribous font leur extraordinaire traversée annuelle alors, l'approvisionnement en cette viande appréciée est pénible et peu abondant. Des incendies de taïga (forêt dense ou ouverte) avaient déjà chassé le petit gibier. Les fruits d'automne n'ont pas permis de généreuses cueillettes, non plus. En situation de faibles réserves carnées, il faudra demeurer plus longtemps sur la carapace glacielle du lac pour, par la pêche blanche, attraper la quantité complémentaire de poisson. Des biens de consommation moins variés défavorisent la qualité nutritive; on devra concocter des bouillons de plantes afin de se prémunir du scorbut.

Il y a bien d'autres ennuis. Une mauvaise manœuvre fait perdre du matériel dans un gros rapide et cause l'avarie du canot de secours. Aux tentes, 
le garçon ne se porte pas bien et Simon doit endurer des rhumatismes que la médecine naturelle ne soulage guère. Surtout, il se produit un drame chez les Shécapio par le décès de l'enfant qui n'avait d'ailleurs jamais été bien depuis sa naissance ; la terre n'étant pas encore profondément gelée, le corps peut être déposé au petit cimetière dominant la terrasse du Camp du Caribou ; le reste de la peinture que l'on avait préservée du gel sert à marquer sur une planche étroite le nom du bébé d'un an. Cet événement malheureux, même un peu attendu, cause une peine qui accentue encore le malaise envahissant le groupe. Bref, la deuxième année qui avait connu moins d'efforts et subi moins de pressions que la précédente se termine dans l'inquiétude mais elle ne fait pas souffrir de la faim.

\section{L'AN III}

La situation est cependant assez sérieuse pour qu'une décision commune soit prise de ne pas prolonger le séjour nordique par un troisième hiver ; on reviendra à Mérosro au prochain automne. Après quelques mois, soit en avril de l'an III, on commence à fermer le camp, départager ce que l'on laisse aux successeurs et ce qu'il faut apporter pour le long voyage de retour ; en principe, ce dernier sera moins difficile, la navigation allant surtout s'effectuer dans le sens du courant. Comme on l'avait fait à Mérosro, on s'exerce à paqueter les effets afin qu'ils voyagent bien et que soient facilement accessibles les articles nécessaires aux nombreux camps d'arrêt. Le jour du départ approchant, on brûle des déchets, mais sans détruire les petites installations pouvant être utiles aux passants ; le matériel qui demeure est entreposé dans des caches légèrement empierrées et recouvertes de petites talles de conifères suggérant un paysage naturel ; qui connaîtra le code ou aura assez de perspicacité les retrouvera. On s'impose un exercice de départ pour vérifier la répartition et le balancement des charges dans les embarcations. Le séjour au Camp du Caribou qui dure depuis vingt mois se terminera le lendemain à l'aurore.

Une fois dans les canots sur le Mushuau, chacun dirige un dernier regard vers la nouvelle croix blanche, comme pour emporter aux gens du village d'en-bas l'âme de l'enfant disparu au Grand Nord.

Presque à mi-distance du voyage de retour, au sud du plateau lacustre central, l'équipe Wawati-Shécapio-Bellefleur rencontre une flottille d'Indiens arrivant du Petit-Mécatina, affluent du golfe du Saint-Laurent, soit une distance encore plus grande que celle de Mérosro; étant à leur deuxième année de voyagement, ils comptent arriver au paradis de la viande dans quelques semaines et y passer au moins un hiver. Aux arrivants, les partants donnent des informations concernant les deux planchers près des rives du Mushuau, les migrations fantaisistes du gros mammifère, le comptoir extrême de Djouac-jjuaq et les caches qui contiennent entre autres une dizaine de peaux 
d'habitation. En échange, ils reçoivent des nouvelles du Sud par le système courant du mocassin-télégraphe. Les partants s'engagent à transporter avec attention les lettres destinées aux Indiens du Fleuve. Puis ils reprennent leur descente vers le sud en récupérant le moteur déposé dạs une cache au moment de la panne sèche lors de la montée.

À Mérosro même, durant la longue absence de Simon et de Catherine, leur maison du village est, comme convenu, occupée par une jeune famille en besoin d'habitat. Au retour du Nord, avant de trouver une solution au relogement du locataire, les deux ménages coexistent sous le même toit.

Par ailleurs, c'est tout le village qui, organisant un mokoshan dans le shaputuan communautaire, reçoit les voyageurs Wawati-Shécapio-Bellefleur et surtout la fillette née au lointain. On s'attriste cependant du décès d'un enfant, même si on ne l'a jamais connu. Des conversations presque sans interruption occupent un bon nombre de jours. La fin de l'année III est bien douce car les pèlerins du Grand Voyage sont revenus parmi les leurs. Sans rapport direct avec sa récente aventure nordique, Simon, par consensus, est choisi chef de la Réserve, motif de fierté pour Catherine et les enfants. Suivant l'espacement pragmatique des canotages à long cours, on ne prévoit pas que quelqu'un du village parte pour le Mushuau, au cours des prochaines années.

Les Grands Voyages, qu'ils soient de l'Ouest, du Nord ou de l'Est, remuent l'âme du participant alors que les observateurs de l'extérieur y identifient quatre traits généraux.

D'abord, tout long déplacement se nourrit d'une immense espérance culturelle. Les Indiens se souhaitent d'entreprendre au moins un tel circuit dans leur vie, d'avoir un enfant né là-bas et, le cas échéant, de participer à de grandes chasses rituelles. L'émotion du participant rappelle les aurores boréales qui, la nuit, embrasent les cieux. Les dits des Anciens et des Shamans font que les membres de la communauté ne s'échappent pas de ces horizons merveilleux qui embaument le quotidien.

En deuxième lieu, le Grand Voyage au caribou représente comme un pèlerinage aux lieux saints dans les grandes religions. Préparé longtemps à l'avance, le déplacement exceptionnel baume tous les sacrifices nécessaires. Le geste est un accomplissement qui marque chaque participant. Les familles qui réalisent ainsi le maximum de leur genre de vie développent le sentiment d'avoir réussi leur existence.

Un aspect majeur confirme l'appartenance d'un peuple, comme conséquence de tout type de parcours. Même sans argumentation proprement géopolitique, les courageux canotages en toutes directions marquent le 
territoire de ceux qui les entreprennent. L'empreinte paysagique est sans doute très légère mais les nations européennes d'occupation se contenteront de bien moins pour réclamer les mêmes terres. Pour ceux qui aiment à donner du sens aux événements singuliers, les pérégrinations par intervalles sur plusieurs centaines de kilomètres, depuis un repeuplement animal millénaire, correspondent à une communication avec les esprits du monde et un dialogue entre les générations. Voilà la façon et la raison les moins effaçables d'une autochtonité antérieure, base très signifiante de l'autochtonité d'aujourd'hui. Ces vagabondages établissent une relation d'intimité et de fidélité par l'usufruit de la terre. Étant donné l'éloquence inhérente à ce titre d'appartenance, les Autochtones n'avaient pas cru bon de le faire reconnaître devant notaire ; aussi, aujourd'hui, pensent-ils que les non-Autochtones, qui avaient le moins droit à ces espaces, s'en sont attribués le plus.

Enfin, les Grands Voyages, vus de la vallée du Saint-Laurent, prennent tous du septentrion. Ils démontrent ainsi une certaine conception de la territorialité en liant le sud et le nord du Québec-Labrador; par le fait, les Indiens posent les premiers gestes de l'unité spatiale de la péninsule ${ }^{16}$.

16. Remerciements : à Huguette Bouchard, Études amérindiennes de Chicoutimi, Léo-Paul Hébert, cégep de Joliette, José Mailhot, anthropologue de Montréal, ainsi qu'au Laboratoire de cartographie du département de Géographie de l'université Laval. 